\title{
Otimização de Propriedades Mecânicas de Misturas Ternárias de Polipropileno (PP)/Borracha de Etileno-Propileno-Dieno (EPDM)/Pó de Pneus (SRT) sob Tração e Impacto usando a Metodologia da Superfície de Resposta (MSR)
}

\author{
Helson M. da Costa, Valéria D. Ramos, Wilson S. da Silva \\ Departamento de Materiais, UERJ \\ Alex da Silva Sirqueira \\ Centro Universitário da Zona Oeste
}

\begin{abstract}
Resumo: A metodologia da superfície de resposta (MSR) é uma coleção de técnicas estatísticas e matemáticas para desenvolver, melhorar e otimizar processos. Neste estudo, a técnica MSR foi aplicada na investigação do comportamento mecânico de diferentes misturas ternárias de PP/EPDM/SRT. Após a mistura apropriada em uma extrusora de dupla rosca co-rotante e a moldagem por injeção, as propriedades mecânicas (resistência à tração e a resistência ao impacto) foram determinadas e usadas como variáveis de resposta. A microscopia eletrônica de varredura (MEV) foi usada para investigar a morfologia das diferentes misturas e interpretar os resultados. Com ferramentas estatísticas específicas, um número mínimo de experimentos permitiu o desenvolvimento de um modelo de superfície de resposta e a otimização das concentrações dos componentes de acordo com o desempenho mecânico. Valores elevados de resistência ao impacto são alcançados $\left(>80 \mathrm{~J} \cdot \mathrm{m}^{-1}\right)$ quando, de acordo com as condições experimentais estudadas, a mistura física de PP/EPDM/SRT mantém as proporções de EPDM e SRT em torno de $25 \%$.
\end{abstract}

Palavras-chave: $M S R, P P, E P D M$, pó de pneu.

\section{Optimization of Mechanical Properties of Polypropylene (PP)/Ethylene-Propylene-Diene Monomer Rubber (EPDM)/Scrap Rubber Tire (SRT) Ternary Mixtures Under Tensile and Impact using the Response Surface Methodology (RSM)}

\begin{abstract}
The response surface methodology (RSM) is a collection of statistical and mathematical techniques for developing, improving and optimizing processes. In this study, RSM technique was applied to the investigation of the mechanical behavior of different PP/EPDM/ SRT ternary mixtures. After appropriate processing in a co-rotating twin extruder and injection molding, the mechanical properties, such as tensile strength and impact strength, were determined and used as response variables. Scanning electron microscopy (SEM) was used for investigating the morphology of the different blends and to interpret the results. With specific statistical tools, a minimum number of experiments allowed the response surface model to be developed and the concentrations of the components to be optimized according to the mechanical performance. High values of impact strength were reached (>80 J.m $\left.\mathrm{m}^{-1}\right)$ when the PP/EPDM/SRT mixture had a EPDM/ SRT ratio of around $25 \%$.
\end{abstract}

Keywords: RSM, PP, EPDM, scrap rubber tire.

\section{Introdução}

O polipropileno (PP) é uma resina que oferece um bom equilíbrio de propriedades térmicas, químicas e elétricas, acompanhadas de uma resistência mecânica moderada. Além disso, apresenta facilidade para incorporação de teores elevados de cargas e agentes de reforço e capacidade de produzir misturas com outros polímeros, incluindo borrachas, o que o torna extremamente versátil como material. Tanto os tipos de PP reforçados como os não-reforçados são aplicados em automóveis; aparelhos domésticos e elétricos; potes e copos para a indústria alimentícia; caixas para embalagens industriais; filmes; entre outras aplicações. Embora apresente uso crescente na indústria automobilística, o comportamento frágil com relação à resistência ao impacto em baixas temperaturas é um fator limitante para maiores aplicações. A fim de melhorar tal desempenho, extensa pesquisa vem sendo desenvolvida através de misturas de PP com elastômeros, em particular, a borracha butílica (BR), a borracha de estireno-butadieno (SBR) e a borracha de etileno-propilenodieno não conjugado (EPDM), as quais possuem valores de $T_{g}$ muito baixos. Há ainda técnicas de polimerização in situ com monômeros específicos para o aumento da resistência ao impacto do $\mathrm{PP}^{[1-9]}$.

Os resíduos de borracha oriundos de pneus inservíveis tornaram-se um passivo ambiental de grande preocupação. A adição de resíduos de pneus moídos (SRT) ou desvulcanizados em composições poliméricas é apresentada na literatura como uma maneira de gerar misturas de fácil processamento, vantajosas economicamente e com aumento da tenacidade da matriz termoplástica de interesse ${ }^{[9-12]}$. Porém, na literatura, o estudo das diferentes misturas entre o PP e borrachas é conduzido de forma aleatória, ou seja, um grande número de composições diferentes

Autor para correspondência: Autor para correspondência: Helson M. da Costa, Departamento de Materiais, Instituto Politécnico, Universidade do Estado do Rio de Janeiro - UERJ, Rua Alberto Rangel, sn, Vila Nova, CEP 28630-050, Nova Friburgo, Rio de Janeiro, RJ, Brasil, e-mail: hmcosta@iprj.uerj.br 
é apresentado e não há uma metodologia definida. Assim, em muitos casos, há um grande gasto de tempo e custos indiretos de experimentação. Desta forma, neste trabalho, o objetivo é introduzir uma combinação da análise de variância e a metodologia da superfície de resposta (MSR) para o estudo de misturas ternárias de PP/EPDM/SRT. Usando-se as propriedades mecânicas (resistência à tração e resistência ao impacto) como variáveis de resposta e a microscopia eletrônica de varredura (MEV) para o estudo da morfologia, as proporções dos componentes (PP, EPDM e SRT) foram otimizadas com um número mínimo de experimentos. Além disso, o mapeamento da superfície de resposta dentro de uma região de interesse foi feito, o que permitiu predizer as mudanças nas propriedades para uma extensa faixa de diferentes composições possíveis.

\section{Experimental}

\section{Materiais}

O polipropileno (PP), homopolímero comercial TS-6100, foi generosamente doado pela empresa Quattor Petroquímica S.A., com índice de fluidez de 16,0 g/10 min (ASTM D1238, $230{ }^{\circ} \mathrm{C}$, $2,16 \mathrm{~kg})$ e gravidade específica de $905 \mathrm{~kg} \cdot \mathrm{m}^{-3}$. A borracha de etileno-propileno-dieno não conjugado (EPDM) foi fornecida pela empresa Branco Indústria e Comércio Ltda. O pó de pneu (SRT) de granulometria aproximada de 40 mesh $(\cong 400 \mu \mathrm{m})$, tipo AG40, foi fornecido pela empresa ArtGoma do Brasil Ltda. O SRT é obtido de pneus inteiros após separação dos resíduos metálicos e de poliéster. A composição aproximada do SRT é: $40-55 \%$ em borracha (principalmente, o copolímero estireno-butadieno SBR; borracha natural - NR; e, polibutadieno - BR), 10-15\% em compostos extraídos em acetona, 25-40\% em negro de fumo e 3-6\% em cinzas. O antioxidante Irganox B215, gentilmente doado pela BASF S.A., foi empregado durante o processamento para evitar degradação das misturas. Para fins de comparação, a mistura PP/ EPDM/SRT 50/25/25\% foi refeita usando-se o pó de pneu (SRT) peneirado em menor granulometria ( 60 mesh ou $\cong 250 \mu \mathrm{m})$ e com adição de $0,5 \%$ de peróxido de dicumila (DCP).

\section{Planejamento das misturas}

Os planejamentos experimentais para o estudo de misturas apresentam uma importante diferença em relação aos planejamentos usuais. Considerando as diferentes variáveis experimentais (condições de processamento, condições de injeção dos corpos de prova, etc.) mantidas constantes, as propriedades finais de uma mistura são determinadas pelas proporções de seus ingredientes, e não por valores absolutos. A soma das proporções dos diversos componentes de uma mistura é sempre $100 \%$. Para uma mistura qualquer de $q$ componentes, podemos escrever (Equação 1):

$$
\sum_{i=1}^{q} x_{i}=100 \%=1
$$

onde $\mathrm{x}_{i}$ representa a proporção do $i$-ésimo componente. Esta equação retira um grau de liberdade das proporções. Para especificar a composição da mistura, só é necessário fixar as proporções de q-1 componentes. A proporção do último componente serás sempre o que falta para completar $100 \%$.

Para sistemas com três fatores independentes, todas as composições possíveis da mistura ternária são representadas pelos pontos pertencentes a um triângulo equilátero. Os vértices correspondem aos componentes puros e os lados às misturas binárias, enquanto os pontos situados no interior do triângulo representam as misturas de três componentes.
O programa MINITAB $15.0^{\mathrm{TM}}{ }^{\mathrm{B}}$ foi usado no planejamento das misturas, análise dos dados das propriedades mecânicas e o procedimento de otimização dos componentes. Neste trabalho, PP, EPDM e SRT foram representados pelas variáveis de entrada, para o sistema em estudo, designadas por $\mathrm{x}_{1}, \mathrm{x}_{2}$ e $\mathrm{x}_{3}$, respectivamente. Além disso, o planejamento foi conduzido de maneira a cobrir apenas uma região dentro daquela possível pelo triângulo de composições, ou seja, foram criadas condições de restrição: $0,50 \leq \mathrm{x}_{1} \leq 1,0$; $0,0 \leq x_{2} \leq 0,25$; e, $0,0 \leq x_{3} \leq 0,25$. A Figura 1 apresenta, através das linhas tracejadas em negrito, a região de interesse do estudo. Os treze círculos negros representam as misturas de PP/EPDM/ SRT que devem ser preparadas para se obter uma superfície de resposta apropriada usando-se uma equação polinomial de grau $n$. As misturas definidas pelo programa MINITAB $15.0^{\mathrm{TM} \otimes}$ são dadas na Tabela 1.

\section{Preparação das misturas e ensaios mecânicos}

PP, EPDM e SRT foram misturados em um misturador rotatório por um período de cinco minutos. Para a extrusão das misturas PP/ EPDM/SRT uma extrusora de dupla rosca co-rotante (Extrusão Brasil) foi usada. A extrusora apresenta cinco zonas de controle de temperatura e um dispositivo de medição de pressão. PP puro e as diferentes misturas foram preparadas com um perfil de temperatura

\section{Planejamento das misturas ternárias PP/ EPDM/ SRT}

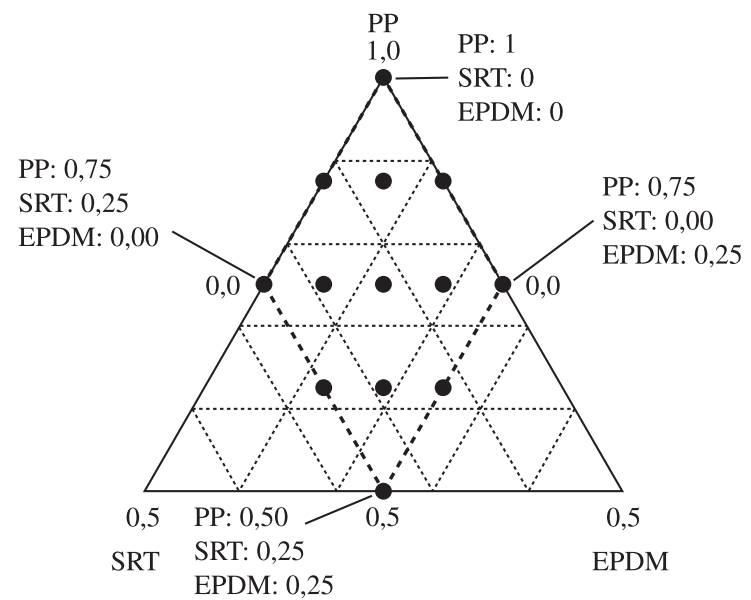

Figura 1. Triângulo de composições para as misturas PP/EPDM/SRT.

Tabela 1. As treze misturas de PP/EPDM/SRT projetadas.

\begin{tabular}{cccc}
\hline Mistura & \multicolumn{3}{c}{ Proporção dos componentes } \\
\cline { 2 - 4 } & $\mathbf{P P}\left(\mathbf{x}_{\mathbf{1}}\right)$ & $\mathbf{E P D M}\left(\mathbf{x}_{\mathbf{2}}\right)$ & $\mathbf{S R T}\left(\mathbf{x}_{\mathbf{3}}\right)$ \\
\hline 1 & 1,000 & 0,000 & 0,000 \\
2 & 0,875 & 0,125 & 0,000 \\
3 & 0,875 & 0,000 & 0,125 \\
4 & 0,875 & 0,0625 & 0,0625 \\
5 & 0,750 & 0,250 & 0,000 \\
6 & 0,750 & 0,000 & 0,250 \\
7 & 0,750 & 0,1875 & 0,0625 \\
8 & 0,750 & 0,0625 & 0,1875 \\
9 & 0,750 & 0,125 & 0,125 \\
10 & 0,625 & 0,250 & 0,125 \\
11 & 0,625 & 0,125 & 0,250 \\
12 & 0,625 & 0,1875 & 0,1875 \\
13 & 0,500 & 0,250 & 0,250 \\
\hline
\end{tabular}


de $180 / 200 / 210 / 220 / 230{ }^{\circ} \mathrm{C}$. A velocidade do dosador na zona de alimentação foi de $8 \mathrm{rpm}$. A velocidade de rotação durante o processamento foi mantida em $100 \mathrm{rpm}$. O extrudado foi resfriado em água fria e, posteriormente, granulado para a injeção dos corpos de prova de resistência à tração e resistência ao impacto.

A moldagem por injeção foi conduzida em uma injetora Battenfeld Plus 35/75, a qual possui três zonas de aquecimento que foram mantidas em $200{ }^{\circ} \mathrm{C}$. As condições experimentais utilizadas foram: velocidade do parafuso, 150 rpm; pressão de injeção, 120 bar; tempo de injeção, 5 segundos; tempo de resfriamento, 40 segundos.

Após condicionamento por 24 horas, as propriedades mecânicas foram avaliadas. Os dados de tensão-deformação foram obtidos através de uma máquina de ensaios universal, Shimadzu AG-I, usando-se corpos de prova do tipo V de acordo com a norma ASTM D-638. A velocidade do ensaio de resistência à tração foi de $50 \mathrm{~mm} /$ min para todas as misturas. A resistência ao impacto Izod dos corpos de prova com entalhe do tipo V foi obtida segundo a norma ASTM D-256. A temperatura foi mantida em $25 \pm 1{ }^{\circ} \mathrm{C}$ para ambos os ensaios mecânicos. $\mathrm{O}$ valor médio e o desvio padrão dos resultados obtidos foram calculados usando-se pelo menos dez corpos de prova de cada mistura.

\section{Análise da superfície de fratura}

A microscopia eletrônica de varredura (MEV) possibilitou a análise da morfologia das misturas poliméricas PP/EPDM/SRT. O exame da superfície de fratura dos corpos de prova após o ensaio de resistência à tração foi conduzido em microscópio eletrônico de varredura (MEV), modelo JEOL (JEOL-6610LV) com voltagem de aceleração de elétrons de 10 a 15 kV.

\section{Resultados e Discussão}

As propriedades mecânicas de resistência à tração e resistência ao impacto foram avaliadas para as diferentes misturas ternárias especificadas pela Tabela 1. Usando-se a análise de variância gerada pelo programa MINITAB $15.0^{\mathrm{TM}} \circledast$, algumas equações polinomiais foram testadas para a construção das superfícies de resposta. $\mathrm{O}$ modelo quadrático especial para três componentes (Equação 2) foi escolhido porque produziu a melhor regressão para os resultados experimentais obtidos.

$$
\begin{aligned}
& \Psi=\alpha_{1} \cdot \mathrm{x}_{1}+\alpha_{2} \cdot \mathrm{x}_{2}+\alpha_{3} \cdot \mathrm{x}_{3}+\alpha_{12} \cdot \mathrm{x}_{1} \mathrm{x}_{2}+\alpha_{13} \cdot \mathrm{x}_{1} \mathrm{x}_{3}+ \\
& +\alpha_{23} \cdot \mathrm{x}_{2} \mathrm{x}_{3}+\alpha_{1123} \cdot \mathrm{x}_{1}{ }^{2} \mathrm{x}_{2} \mathrm{x}_{3}+\alpha_{1223} \cdot \mathrm{x}_{1} \mathrm{x}_{2}{ }^{2} \mathrm{x}_{3}+\alpha_{1233} \cdot \mathrm{x}_{1} \mathrm{x}_{2} \mathrm{x}_{3}{ }^{2}
\end{aligned}
$$

onde $\Psi$ é o dado da variável de resposta; $\alpha_{1}, \alpha_{2}$ etc. são os coeficientes do polinômio; e, $\mathrm{x}_{1}, \mathrm{x}_{2}$ e $\mathrm{x}_{3}$ são variáveis de entrada para o sistema em estudo e representam as proporções de PP, EPDM e SRT, respectivamente, na mistura ternária. A Equação 2 foi usada para a construção das superfícies de resposta apresentadas nas Figuras 2-6.

\section{Propriedades em tração}

$\mathrm{Na}$ Figura 2, a resistência máxima à tração das misturas PP/ EPDM/SRT é mostrada. Pode ser observado que a resistência máxima das misturas ternárias diminui com o aumento da concentração de EPDM ou SRT. Somente quando o conteúdo de PP é maior do que $85 \%$ na mistura é que a resistência máxima aproxima-se de valores superiores a $30 \mathrm{MPa}$ - a pequena área em negro representada no triângulo da Figura 2.

$\mathrm{Na}$ literatura ${ }^{[10-12]}$ é reportado que a adição de SRT na forma de carga particulada ao $\mathrm{PP}$ reduz a resistência à tração e o alongamento na ruptura como consequência da fraca adesão entre as fases e a concentração de tensões ao redor das partículas de SRT. SRT apresenta uma pobre capacidade de suportar a transferência de tensões a partir da matriz de PP e, portanto, as misturas ternárias ricas em SRT exibem uma diminuição acentuada desta propriedade.

Investigações similares de Ishak e Bakar ${ }^{[13]}$ e Plawky et al. ${ }^{[14]}$ sugerem que a adição de EPDM as misturas PP/EPDM/SRT pode aumentar a adesão entre a matriz de PP e os domínios de SRT através de modificação da interface. Ainda que isso possa estar ocorrendo, tal hipótese não foi suficiente para a manutenção dos valores de resistência em um patamar elevado. Em princípio, valores críticos, de acordo com o modelo matemático proposto, são alcançados em composições próximas ao vértice inferior mais baixo da região de interesse mostrada na Figura 1 - proporções de PP/EPDM/SRT de $50 / 25 / 25 \%$, respectivamente.

Na Figura 3, o alongamento na ruptura para as misturas ternárias é apresentado. O comportamento observado para as misturas é similar àquele encontrado na resistência máxima à tração, ou seja, a adição de pó de pneu ao polipropileno não resulta em melhoria. Baixos valores de alongamento na ruptura $(<75 \%)$ são encontrados em misturas ternárias ricas em SRT, as quais podem ser vistas na grande região em negro que aparece no triângulo da Figura 3. As partículas de SRT não exercem o papel de agentes de transferência de energia na mistura. Se não há adesão, o descolamento das partículas de SRT da matriz e a formação de vazios que irá ocorrer na interface ajudam na maior propagação de trincas no ensaio de resistência à tração ${ }^{[15-18]}$. Composições onde o conteúdo de EPDM é similar ou superior àquele de SRT mostram alongamento na ruptura na faixa de 75 até $150 \%$ - pequena região em branco no triângulo de composições da Figura 3. Aparentemente, o EPDM desenvolve alguma melhoria na falta de adesão entre duas fases distintas (SRT e PP). De acordo com Phadke e De ${ }^{[10]}$, o EPDM pode estabilizar a distribuição do pó de pneu na matriz de PP.

A tenacidade corresponde à capacidade que o material apresenta de absorver energia até a fratura ${ }^{[19]}$. Neste trabalho, a tenacidade foi estimada através da integração da área sob a curva tensãodeformação. A Figura 4 apresenta os resultados experimentais referentes à tenacidade para as diferentes misturas PP/EPDM/SRT.

A mistura direta de plásticos com borrachas tem sido largamente usada como forma de melhoria da tenacidade, ainda que reduza a

Máxima resistência à tração (MPa)

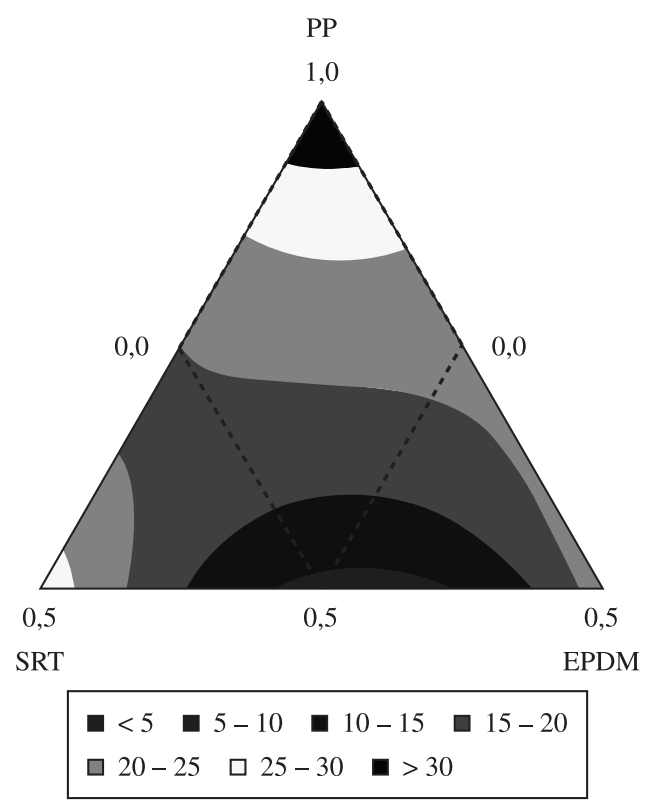

Figura 2. Resistência máxima à tração das composições de PP/EPDM/SRT. 
Alongamento na ruptura (\%)

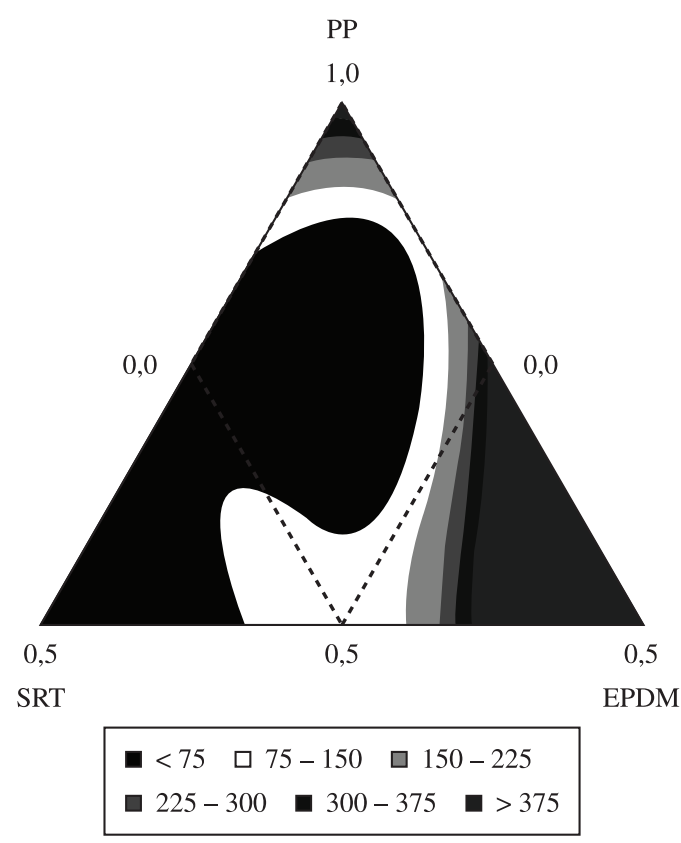

Figura 3. Alongamento na ruptura das composições de PP/EPDM/SRT.

Tenacidade (J)

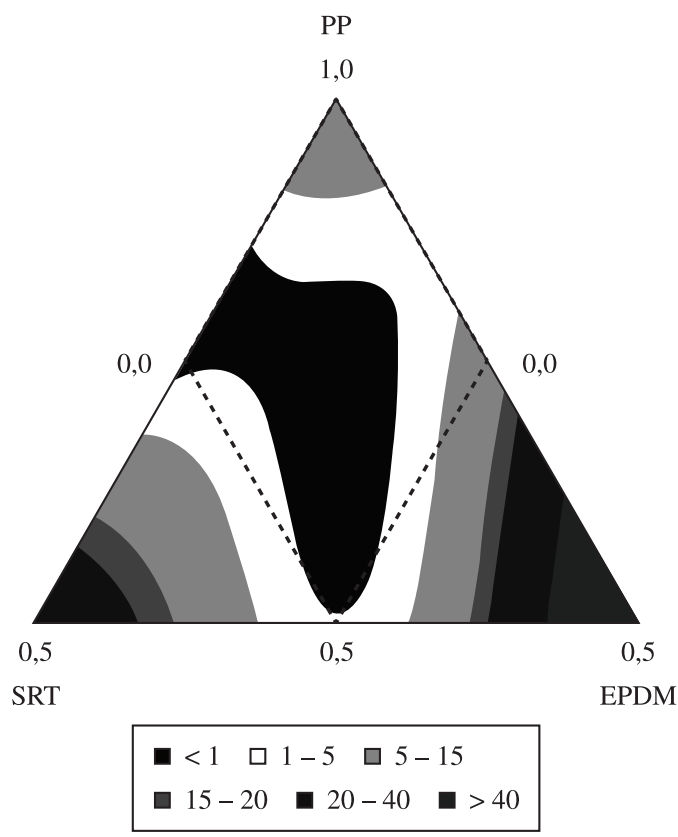

Figura 4. Tenacidade das composições de PP/EPDM/SRT.

rigidez dos materiais ${ }^{[15-20]}$. Ao mesmo tempo, é encontrado em muitos trabalhos na literatura que os fatores mais importantes no aumento de tenacidade em PP através do uso de borrachas são: i) conteúdo de borracha; ii) tamanho e distribuição das partículas de borracha; iii) grau de ligações cruzadas; iv) grau de adesão interfacial; e, v) tamanho e formato dos esferulitos de $\mathrm{PP}^{[20-22]}$. A tenacidade das misturas de $\mathrm{PP} / \mathrm{EPDM} / \mathrm{SRT}$ diminui progressivamente quando o percentual de SRT aumenta (área em negro na Figura 4 onde os valores de tenacidade estão abaixo de $1 \mathrm{~J}$ ). Sem dúvida, as partículas de SRT mostram fraca adesão
Módulo de elasticidade (MPa)

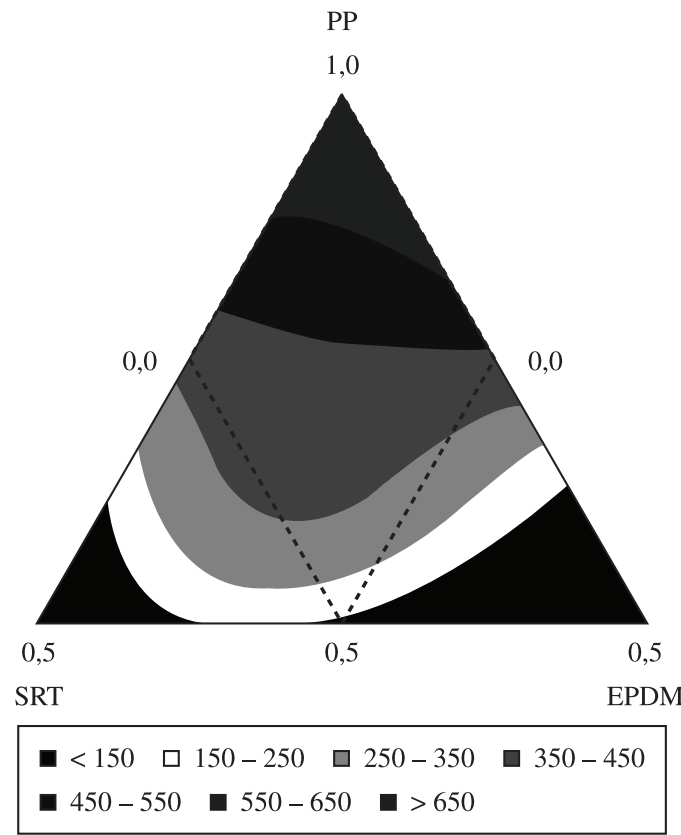

Figura 5. Módulo elástico das composições de PP/EPDM/SRT. Resistência ao impacto (J.m-1)

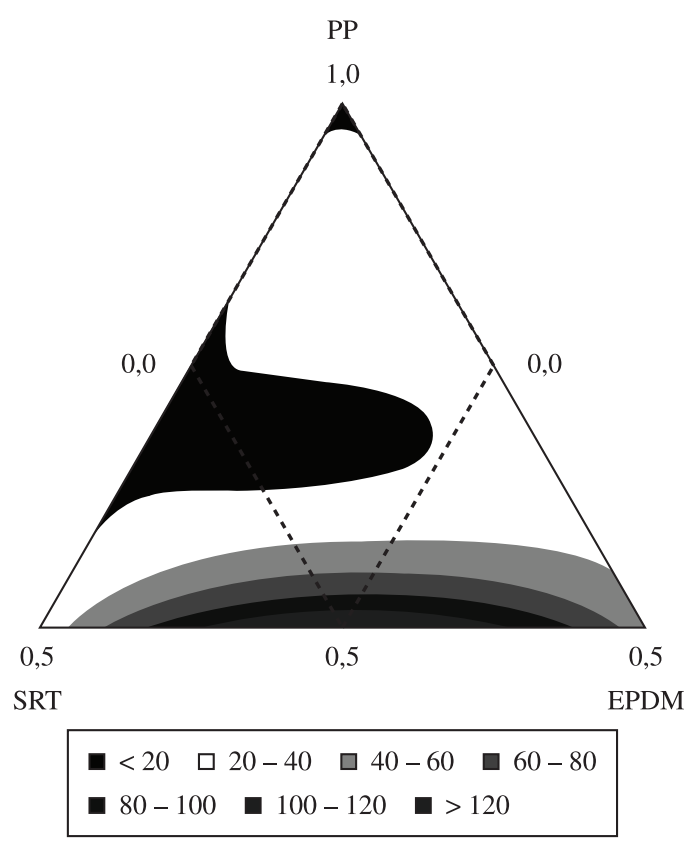

Figura 6. Resistência ao impacto das composições de PP/EPDM/SRT.

à matriz de PP e microvazios na interface devem se desenvolver, o que resulta em uma diminuição das propriedades mecânicas. Geralmente, a adição de um terceiro componente ou um compatibilizante em uma mistura binária é conduzida de forma a se obter uma mistura mais homogênea e uma morfologia da fase secundária mais adequada ${ }^{[18-22]}$. Essa observação pode ser considerada verdadeira quando o EPDM é usado - região em branco com formato de $\mathrm{L}$ invertido situada no interior do triângulo da Figura 4, onde os valores de tenacidade apresentam discreto aumento situando-se entre 1 e $5 \mathrm{~J}$. 


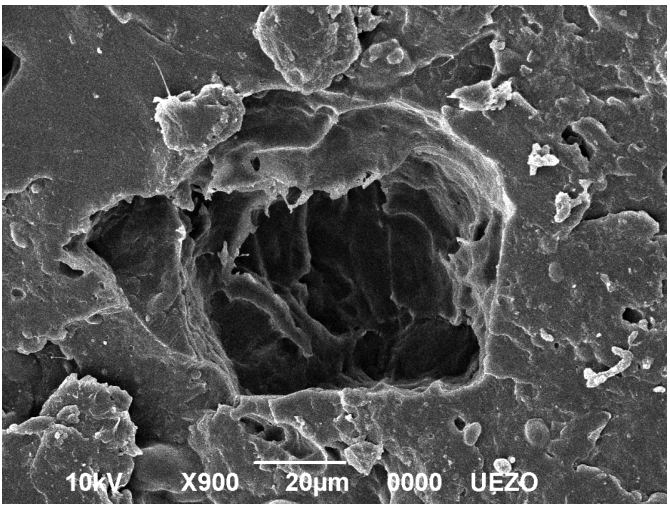

(a)

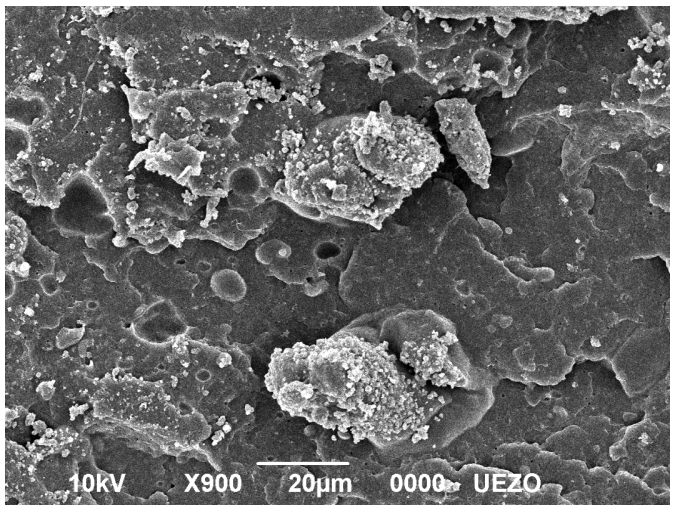

(c)

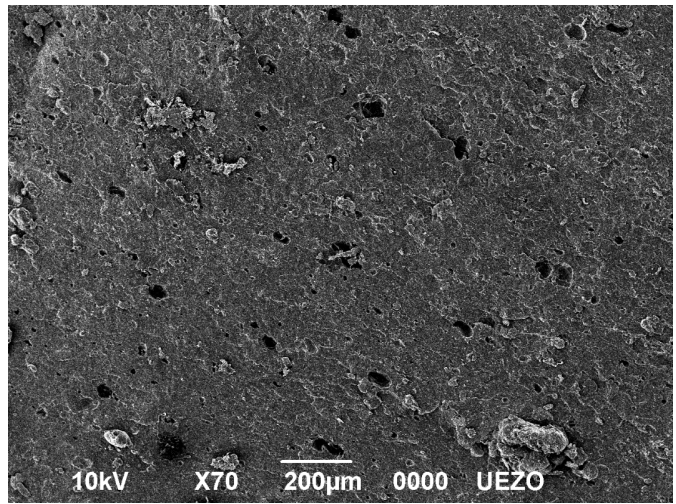

(d2)

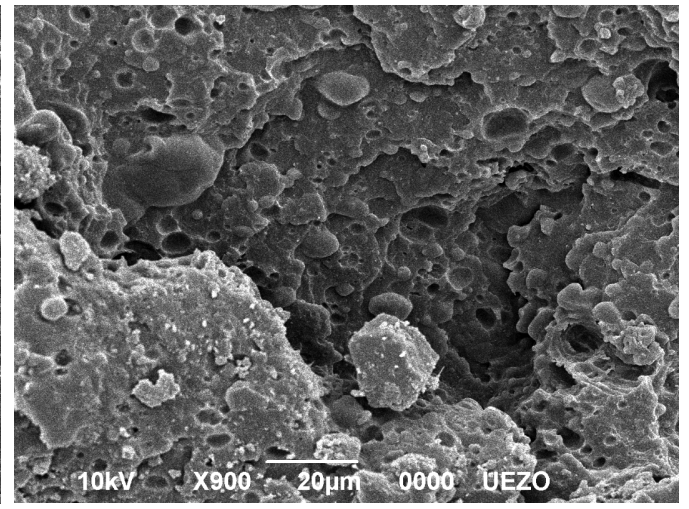

(b)

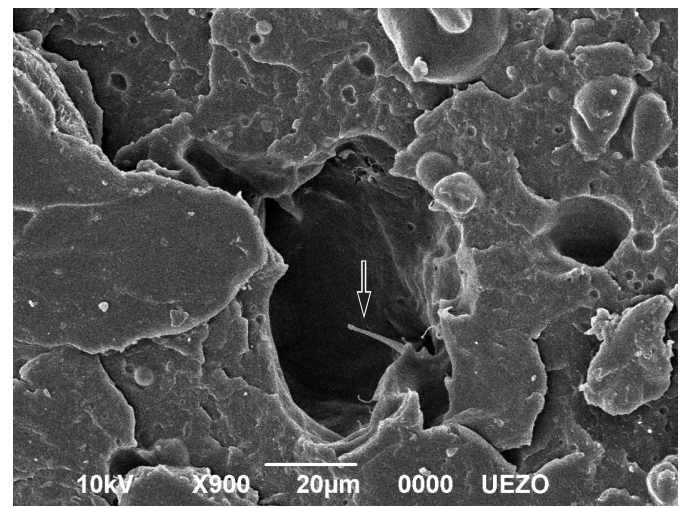

(d1)

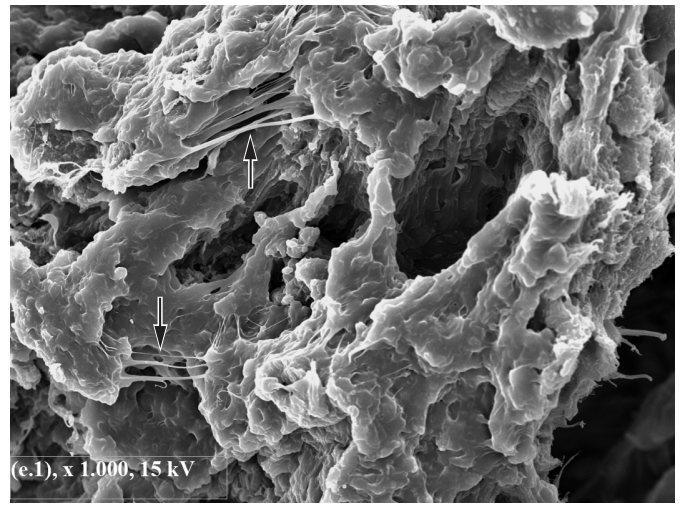

(e1)

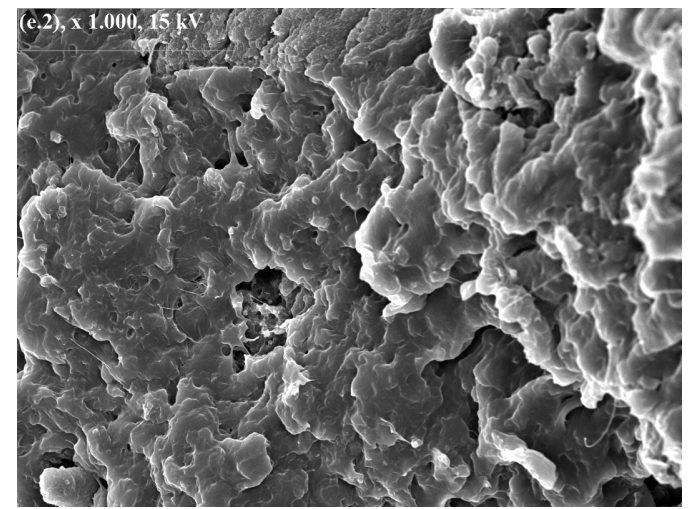

(e2)

Figura 7. Micrografias das superfícies de fratura dos corpos de prova de tração das misturas de PP/EPDM/SRT. a) PP/EPDM/SRT 75/0/25\%; b) PP/EPDM/ SRT 75,00/6,25/18,75\%; c) PP/EPDM/SRT 62,50/18,75/18,75\%; d.1) PP/EPDM/SRT 50/25/25\%; d.2) PP/EPDM/SRT 50/25/25\%, superfície tratada com $\mathrm{HNO}_{3} 10 \%$; e) PP/EPDM/SRT 50/25/25\%, composição contendo 0,1\% de DCP. 
O módulo elástico (E) ou a rigidez das misturas ternárias é mostrado na Figura 5. SRT e EPDM são materiais mais macios do que o PP e, portanto, como esperado, a adição de um ou outro resulta em um decréscimo nos valores de E. Isso pode ser claramente observado na região pontilhada (valores de E entre 450 e $550 \mathrm{MPa}$ ) e na região quadriculada (valores de E entre 350 e $450 \mathrm{MPa}$ ) do triângulo de composições.

\section{Resistência ao impacto}

A resistência ao impacto de termoplásticos é frequentemente aumentada pela incorporação de uma fase elastomérica, isto é, uma fase borrachosa auxilia na tenacificação da matriz. Para a incorporação de resíduos particulados de pneus inservíveis em uma matriz polimérica, o tamanho de partícula da borracha e a adesão entre a matriz e a borracha moída são considerados os fatores principais no desempenho mecânico das misturas ${ }^{[23-26]}$.

Na Figura 6, é mostrado que a adição de resíduos de pneus à matriz de polipropileno (PP) não resulta em um aumento da resistência ao impacto, mas apenas em uma manutenção dos valores

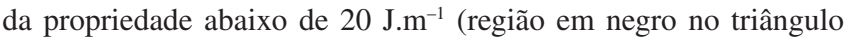
de composições). Tal fato é devido à insuficiente adesão entre as duas fases, como já discutido anteriormente. Porém, quando os percentuais de EPDM e SRT são mantidos próximos a $25 \%$, altos valores de resistência ao impacto são alcançados - regiões pontilhada e quadriculada da Figura 6, onde os valores estão entre 60 e $100 \mathrm{~J} . \mathrm{m}^{-1}$.

Stehling et al. $^{[4]}$ estudaram as dispersões da borracha de poli(etileno-co-propileno) (PEP) e de polietileno de alta densidade (HDPE) em uma matriz de polipropileno (PP). Eles observaram que quando pequenas quantidades de PEP e HDPE são misturadas em $\mathrm{PP}$, há uma tendência ao encapsulamento de partículas de PEP pela fase de HDPE (efeito "casca"). Assim, com pequenos percentuais de HDPE, a mistura apresenta um mecanismo de microfissuramento com características dúcteis acentuadas pela presença de partículas de borracha, o que reflete em uma elevada resistência ao impacto. Em outra investigação, foi observado por Setz et al. ${ }^{[26]}$ que o polipropileno tem boa compatibilidade com os copolímeros em bloco de etileno-butileno (EB). Tal fato contribuiu, segundo os autores, para a melhoria da miscibilidade do PP com PEP.

Talvez, o EPDM apresente igual comportamento nas misturas de PP/EPDM/SRT investigadas. Se o EPDM tende a formar uma casca superficial em torno das partículas de SRT, então, tal cobertura deve produzir uma interface mole e melhor adesão ao PP. Assim, há como resultado líquido uma melhoria na resistência ao impacto da mistura 50/25/25\% de PP/EPDM/SRT.

\section{Microscopia}

A Figura 7 apresenta as micrografias de MEV para a superfície de fratura dos corpos de prova de diferentes misturas submetidos do ensaio de tração. As micrografias das misturas PP/EPDM/ SRT revelam uma superfície morfologicamente típica de misturas de componentes imiscíveis. Contudo, a adição de EPDM produz diferentes características superficiais - uma morfologia de aspecto mais homogêneo e liso do que o observado em composições ricas em SRT. Na Figura 7a, pode ser observado que para a mistura PP/ EPDM/SRT 75/0/25\%, mistura sem EPDM, há grandes domínios e protrusões da fase dispersa presente. Além disso, a micrografia revela os vazios resultantes do descolamento das partículas de SRT da matriz de PP. Essa morfologia revela a fraca adesão entre as fases e explica a pronunciada redução de propriedades mecânicas das composições ricas em SRT.
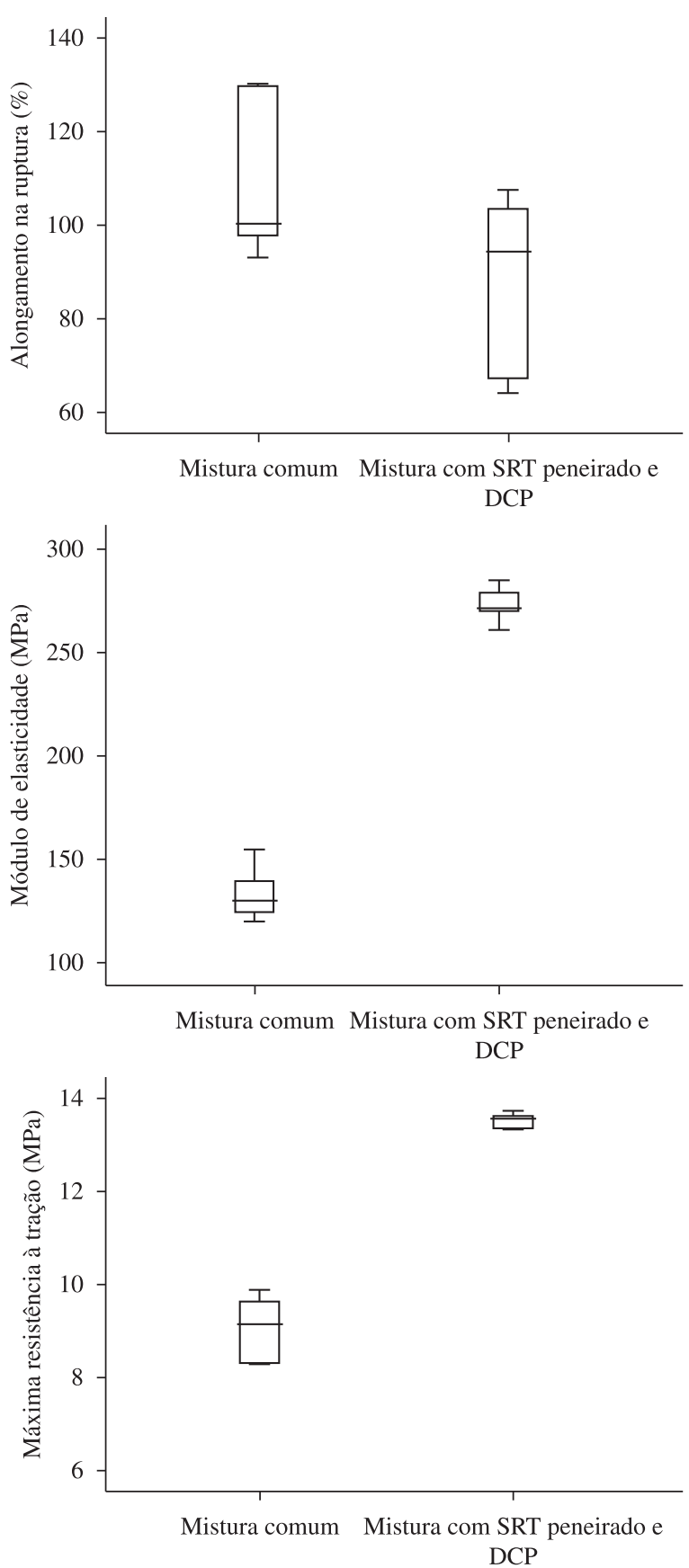

Figura 8. Propriedades mecânicas da mistura PP/EPDM/SRT 50/25/25\% comum e com SRT peneirado e $0,5 \%$ de DCP.

As Figuras 7b,c mostram a matriz de PP das composições contendo 6,25 e 18,75\% de EPDM, respectivamente. O EPDM parece desenvolver um papel "compatibilizante" entre o SRT e a matriz de PP. A morfologia exibe uma redução dos domínios das partículas de SRT e uma menor protrusão é verificada. Tal fato conduz a um aumento das propriedades mecânicas, especialmente a resistência ao impacto.

A Figura 7d.1 representa a mistura PP/EPDM/SRT 50/25/25\%. Nesta mistura ternária, há evidências de deformação plástica ocorrendo na interface entre a matriz de PP e as partículas de SRT - estrutura fibrilar indicada pela seta na Figura 7d.1. A observação de uma estrutura deste tipo pode ser útil na explicação do aumento 
da resistência ao impacto da composição indicada, pois a ocorrência de vazios acompanhados da formação de fibrilas na interface entre a matriz de PP e as fases dispersas indica um mecanismo de cavitação no lugar do simples descolamento das partículas de SRT. Assim sendo, o EPDM parece melhorar a compatibilidade do PP e das partículas de SRT aumentando a adesão interfacial, como sugerem trabalhos similares encontrados na literatura ${ }^{[4,16,26]}$.

A superfície de fratura da composição PP/EPDM/SRT $50 / 25 / 25 \%$ foi tratada com $\mathrm{HNO}_{3} 10 \%$ por 1 hora e reanalisada através de MEV conforme mostra a Figura 7d.2. A morfologia exibe um pequeno número de vazios e uma melhor dispersão quando comparada com a de outras composições. Novamente, tal fato corrobora com as observações já discutidas e explica a melhoria da resistência ao impacto verificada.

Nas Figuras 7e.1,e.2 é mostrada a superfície de fratura do corpo de prova da composição PP/EPDM/SRT 50/25/25\% contendo SRT peneirado em menor granulometria (60 mesh) e $0,5 \%$ de peróxido de dicumila (DCP). Observa-se que o material exibe uma textura mais homogênea, na qual a fase dispersa não pode ser claramente identificada. Há claras evidências de um mecanismo de fratura mais dúctil, ou seja, com uma deformação plástica mais visível ${ }^{[9,11,12]}$. Além disso, a estrutura fibrilar é novamente verificada (Figura 7e.1), porém, desta vez, de forma bem mais acentuada do que o verificado anteriormente para a mesma composição (Figura d.1).

Pela morfologia, há uma indicação de que as propriedades mecânicas da mistura PP/EPDM/SRT 50/25/25\% contendo SRT peneirado em menor granulometria (60 mesh) e 0,5\% de peróxido de dicumila (DCP) sejam superiores à mistura original. Tal fato pode ser comprovado pela Figura 8, o que corrobora o já discutido anteriormente e onde se observa um significante aumento em termos de resistência à tração e módulo de elasticidade. Para a propriedade de resistência ao impacto, o aumento no desempenho foi mais modesto com o valor final alcançado situando-se na região definida entre $100-120$ J.m ${ }^{-1}$ da Figura 6.

\section{Conclusões}

O estudo de misturas poliméricas, particularmente misturas de PP/EPDM/SRT, pode ser desenvolvido usando-se a metodologia da superfície de resposta (MSR). Essa técnica é útil na determinação das quantidades ótimas de cada componente da mistura e na obtenção de equações, as quais podem ser empregadas para mapear uma superfície de resposta sobre uma região de particular interesse. Com as condições experimentais adotas e as ferramentas estatísticas (análise de variância e MSR) utilizadas nesta investigação, foi possível concluir que:

i) Com apenas treze experimentos é possível a previsão da composição ótima de uma mistura ternária PP/EPDM/SRT através da obtenção de equações polinomiais de grau apropriado. Além disso, as equações obtidas, embora sejam verdadeiramente úteis apenas dentro da região de interesse, também podem gerar projeções sobre o comportamento mecânico investigado até os extremos inferiores do triângulo de composições apresentado na Figura 1;

ii) As propriedades mecânicas em tração exibem um rápido declínio quando o percentual de partículas de SRT é aumentado nas misturas ternárias. Isto ocorre em função da fraca adesão entre SRT e a matriz de PP. A diminuição da granulometria das partículas de SRT produz um desempenho melhor;

iii) EPDM parece auxiliar na melhoria da adesão interfacial entre PP e as partículas de SRT. Particularmente, a propriedade de resistência ao impacto e as análises de MEV corroboram com esta conclusão. A adição de $0,5 \%$ de DCP contribui para a reticulação da fase elastomérica de EPDM, o que auxilia na retenção ou melhoria do desempenho mecânico; iv) Valores elevados de resistência ao impacto são alcançados $\left(>80 \mathrm{~J}^{-\mathrm{m}^{-1}}\right)$ quando, de acordo com as condições experimentais estudadas, a mistura física de PP/EPDM/SRT mantém as proporções de EPDM e SRT em torno de $25 \%$.

\section{Agradecimentos}

Os autores agradecem ao suporte financeiro providenciado pelos órgãos de fomento CNPq, CAPES e FAPERJ.

\section{Referências Bibliográficas}

1. Xanthos, M. - Polym. Eng. Sci., 28, p.1392 (1988). http://dx.doi. org/10.1002/pen.760282108

2. Utracki, L. A. - "Commercial Polymer Blends", Chapman and Hall, London (1998). http://dx.doi.org/10.1007/978-1-4615-5789-0

3. Jaziri, M.; Mnif, N.; Nageotte, V. M. \& Camby, H. P. - Polym. Eng. Sci., 47, p.1009 (2007). http://dx.doi.org/10.1002/pen.20758

4. Stehling, F. C.; Huff, T.; Speed, C. S. \& Wissler, G. - J. Appl. Polym. Sci., 26, p.2693 (1981). http://dx.doi.org/10.1002/app.1981.070260818

5. Galli, P.; Danesi, S. \& Simonazzi, T. - Polym. Eng. Sci., 24, p.544 (1984). http://dx.doi.org/10.1002/pen.760240807

6. Tjong, S. C.; Li, W. D. \& Li, R. K. Y. - Eur. Polym. J., 34, p.755 (1998). http://dx.doi.org/10.1016/S0014-3057(97)00182-1

7. Yang, H.; Li, B.; Wang, K.; Xie, B.; Yiang, M. \& Hou, M. - Eur. Polym. J., 44, p.113 (2008). http://dx.doi.org/10.1016/j.eurpolymj.2007.10.028

8. Tang, X. G.; Bao, R. Y.; Yang, W.; Xie, B.; Yiang, M. \& Hou, M. - Eur. Polym. J., 45, p.1448 (2009). http://dx.doi.org/10.1016/j.eurpolymj.2009.02.004

9. Yeh, J. T. \& Lin, S. C. - J. Appl. Polym. Sci., 114, p.2806 (2009). http:// dx.doi.org/10.1002/app.30865

10. Phadke, A. \& De, S. K. - Polym. Eng. Sci., 26, p.1079 (1986). http://dx.doi. org/10.1002/pen.760261508

11. Tantayanon, S. \& Juikham, S. - J. Appl. Polym. Sci., 91, p.510 (2004). http://dx.doi.org/10.1002/app.13182

12. Lee, S. H.; Belasubramanian, M. \& Kim. J. K. - J. Appl. Polym. Sci., 106, p.3193 (2007). http://dx.doi.org/10.1002/app.26489

13. Ishak, Z. A. M. \& Bakar, A. A. - Eur. Polym. J., 31, p.259 (1995). http:// dx.doi.org/10.1016/0014-3057(94)00156-1

14. Plawky, U.; Schlabs, M. \& Wening, W. - J. Appl. Polym. Sci., 59, p.1891 (1996). http://dx.doi.org/10.1002/(SICI)10974628(19960321)59:12\%3C1891::AID-APP11\%3E3.0.CO;2-V

15. Fayte, R.; Jerome, R. \& Teyssié, P. H. - Polym. Eng. Sci., 27(5), p.328 (1987).

16. Horák, Z.; Fort, F.; Hlavatá, D.; Lednický, F. \& Vecerka, V. - Polymer, 37, p.65 (1996).

17. Ismail, H.; Nasaruddin, M. N. \& Rozman, H. D. - Eur. Polym. J., 35, p.1429 (1999). http://dx.doi.org/10.1016/S0014-3057(98)00223-7

18. Siriwardena, S.; Ismail, H.; Ishiaku, U. S. \& Perera, M. C. S. - J. Appl. Polym. Sci., 85, p.438 (2002). http://dx.doi.org/10.1002/app.10713

19. Da Costa, H. M.; Ramos, V. D. \& Rocha, M. C. G. - Polym. Test., 25, p.498 (2006).

20. Da Costa, H. M.; Ramos, V. D.; Da Silva, W. S. \& Sirqueira, A. S. - Polym. Test., 29, p.572 (2010). http://dx.doi.org/10.1016/j. polymertesting.2010.04.003

21. Oliphant, K. \& Baker, W. E. - Polym. Eng. Sci., 33, p.166 (1993). http:// dx.doi.org/10.1002/pen.760330307

22. Phinyocheep, P.; Axtell, F. H. \& Laosee, T. - J. Appl. Polym. Sci., 86, p.148 (2002). http://dx.doi.org/10.1002/app.10917

23. Kager-Kocsis, J.; Kalló, A. \& Kuleznev, V. N. - Polymer, 25, p.279 (1984).

24. Zhang, B. Z.; Uhlmann, D. R. \& Vander, J. B. - J. Appl. Polym. Sci., 30, p.2485 (1985). http://dx.doi.org/10.1002/app.1985.070300617

25. Da Costa, H. M. \& Ramos, V. D. - Polym. Test., 27, p.27 (2008). http:// dx.doi.org/10.1016/j.polymertesting.2007.08.001

26. Setz, S.; Stricker, F.; Kressier, J.; Duschek, T. \& Mulhaupt, R. - J. Appl. Polym. Sci., 59, p.1117 (1996). http://dx.doi.org/10.1002/(SICI)10974628(19960214)59:7\%3C1117::AID-APP8\%3E3.0.CO;2-H

Enviado: $02 / 11 / 10$

Reenviado: 10/01/11

Aceito: $15 / 04 / 11$ 